\title{
Prevalência de anomalias de forma e número em pacientes ortodônticos: Estudo
}

\section{observacional}

\author{
Prevalence of anomalies of shape and number in orthodontic patients: Observational study \\ Prevalencia de anomalías de forma y número en pacientes de ortodoncia: Un estudio observacional
}

Recebido: 23/06/2021 | Revisado: 04/07/2021 | Aceito: 09/07/2021 | Publicado: 20/07/2021

\author{
Bianca Xavier da Silva \\ ORCID: https://orcid.org/0000-0002-4127-0264 \\ Universidade Metropolitana de Santos, Brasil \\ E-mail: biancaxaviers@hotmail.com \\ Sabrina Buchmann Rossi \\ ORCID: https://orcid.org/0000-0002-0210-938X \\ Universidade Metropolitana de Santos, Brasil \\ E-mail: sarossi@uol.com.br \\ Rodrigo Alves Ribeiro \\ ORCID: https://orcid.org/0000-0003-3131-3678 \\ Universidade Metropolitana de Santos, Brasil \\ E-mail: xandu@unimes.br \\ Wilson Roberto Sendyk \\ ORCID: https://orcid.org/0000-0002-3742-1330 \\ Universidade Santo Amaro, Brasil \\ E-mail: wsendyk@prof.unisa.br \\ Caio Vinicius Gonçalves Romano-Torres \\ ORCID: https://orcid.org/0000-0001-9864-6894 \\ Universidade Santo Amaro, Brasil \\ E-mail: cvgrt@hotmail.com \\ Marcelo de Melo Quintela \\ ORCID: https://orcid.org/0000-0003-0818-8493 \\ Universidade Metropolitana de Santos, Brasil \\ Universidade Santo Amaro, Brasil \\ E-mail: marceloquintela@uol.com.br
}

\begin{abstract}
Resumo
Anomalias dentárias são resultado de distúrbios que ocorrem no processo de odontogênese - responsável pelo desenvolvimento do órgão dental. Essas alterações geralmente acontecem na fase de formação e diferenciação celular. Sendo assim, o objetivo da presente pesquisa foi avaliar a prevalência de anomalias de forma e número em pacientes ortodônticos da Clínica de Especialização da Universidade Metropolitana de Santos. Foram analisadas 262 radiografias panorâmicas, de indivíduos do gênero masculino e feminino, com idade de 06 a 50 anos, que realizaram ou estão realizando tratamento ortodôntico, acrescentando à análise da radiografia panorâmica e de modelos, a consulta dos prontuários clínicos. Para coleta de dados das anomalias dentárias, foi elaborada uma planilha de dados no programa Microsoft Excel. O teste estatístico não paramétrico utilizado foi Mann-Whitney ( $<<0.05)$. Foi observado um total de 114 anomalias dentárias, sendo a agenesia a anomalia mais frequente, se apresentando em $27,86 \%$ dos pacientes, sendo $30 \%$ apresentada apenas por terceiros molares. A taurodontia é a segunda anomalia mais frequente apresentada por 7,25\% dos casos, sendo 47,37\% do gênero masculino e 52,63\% do gênero feminino. Seguiu-se com os supranumerários acometendo $4,20 \%$, apresentando-se mais em homens $(81,8 \%)$ do que em mulheres $(18,2 \%)$. As anomalias de número foram muito mais prevalentes do que as anomalias de forma, sendo agenesia e taurodontia as mais prevalentes entre pacientes ortodônticos.
\end{abstract}

Palavras-chave: Prevalência; Anomalias dentárias; Radiografias panorâmicas; Ortodontia.

\begin{abstract}
Dental anomalies are the result of disorders that occur in the odontogenesis process - responsible for the development of the dental organ. These changes usually occur in the cell formation and differentiation phase. The objective of the present research was to evaluate the prevalence of anomalies of shape and number in orthodontic patients of the Posgraduation Clinic of Universidade Metropolitana de Santos. It was analyzed 262 panoramic radiographs of male and female individuals aged between 06 and 50 years, who underwent or are undergoing orthodontic treatment, adding to the analysis of panoramic radiography and models, the consultation of clinical records. To collect data on dental anomalies, a data spreadsheet was prepared using Microsoft Excel. The statistical test used was Mann-Whitney (p $<0.05)$. A total of 114 dental anomalies were observed, with agenesis being the most frequent anomaly, presenting in
\end{abstract}


$27,86 \%$ of patients, with $30 \%$ being presented only by third molars. Bullfighting is the second most frequent anomaly presented by $7,25 \%$ of the cases, being $47,37 \%$ male and $52,63 \%$ female. It followed with the supernumeraries affecting $4,20 \%$, presenting more in men $(81,8 \%)$ than in women $(18,2 \%)$. Number anomalies were much more prevalent than shape anomalies, with agenesis and taurodontics being the most prevalent among orthodontic patients.

Keywords: Prevalence; Dental anomalies; Panoramic radiographs; Orthodontics.

\section{Resumen}

Las anomalías dentales son el resultado de trastornos que ocurren en el proceso de odontogénesis, responsable del desarrollo del órgano dental. Estas alteraciones suelen ocurrir en la fase de formación y diferenciación celular. Por lo tanto, el objetivo de esta investigación fue evaluar la prevalencia de anomalías de forma y número en pacientes de ortodoncia en la Clínica de Especialización de la Universidade Metropolitana de Santos. Se analizaron un total de 262 radiografías panorámicas de individuos masculinos y femeninos, de 06 a 50 años, sometidos o en tratamiento de ortodoncia, sumando al análisis de radiografías panorámicas y modelos, la consulta de historias clínicas. Para recopilar datos sobre anomalías dentales, se creó una hoja de datos utilizando el programa Microsoft Excel. La prueba estadística no paramétrica utilizada fue la de Mann-Whitney $(\mathrm{p}<0,05)$. Se observaron un total de 114 anomalías dentarias, siendo la agenesia la anomalía más frecuente, presente en el $27,86 \%$ de los pacientes, siendo el $30 \%$ presentado solo por terceros molares. La taurodoncia es la segunda anomalía más frecuente que presenta el 7,25\% de los casos, con 47,37\% hombres y 52,63\% mujeres. Le siguió con los supernumerarios afectando al 4,20\%, presentándose más en hombres $(81,8 \%)$ que en mujeres $(18,2 \%)$. Las anomalías numéricas fueron mucho más frecuentes que las anomalías de forma, siendo la agenesia y la taurodoncia las más frecuentes entre los pacientes de ortodoncia.

Palabras clave: Prevalencia; Anomalías Dentarías; Radiografías Panorámicas; Ortodoncia.

\section{Introdução}

Anomalias dentárias são resultado de distúrbios que ocorrem no processo de odontogênese - responsável pelo desenvolvimento do órgão dental. Essas alterações geralmente acontecem na fase de formação e diferenciação celular, causando falhas estruturais que podem ser classificadas em congênitas, hereditárias ou adquiridas (Andrade et al., 2017; Martins Neto et al., 2019). Os fatores etiológicos das anomalias podem ser ambientais, genéticos, locais ou sistêmicos (Carneiro, 2014; Torres et al., 2015; Grau, 2016; Bilge et al., 2017). As pesquisas de Carneiro (2014) e Martins Neto et al. (2019) correlacionaram a ocorrência das anomalias a algum fator evolutivo não-elucidado, relacionado à alimentação e mastigação.

A agenesia é a anomalia de desenvolvimento registrada como a mais comum na população mundial, e varia de 3,02\% a 64,2\%, de acordo com Carneiro (2014) e Fournier et al. (2018). A agenesia é caracterizada pela ausência de dentes, podendo ocorrer falta de, apenas, alguns elementos dentários sendo chamada de hipodontia; mais de seis elementos, designada de oligodontia, e quando há ausência completa dos dentes, anodontia. Podem ocorrer agenesias tanto na dentição decídua como na permanente, de maneiras isoladas, como às associadas a síndromes ou em conjunto com outras alterações dentárias (Torres et al., 2015; Santos et al., 2017). Entretanto, quando existe aumento no número de peças dentárias, tais elementos são denominados dentes supranumerários, e variam de 0,28\% a 16,5\% (Carneiro, 2014; Capoani e Gonçalves, 2019), sendo classificados de acordo com a sua localização, de modo que, se estão posicionados na linha mediana são chamados mesiodens, e se em região de molares, designados também distomolar ou paramolar (Torres et al., 2015). A maioria dos relatos de agenesia são de incisivo lateral superior, enquanto os supranumerários também costumam estar mais localizados em regiões da maxila, podendo se apresentar com sua forma modificada (cônico) ou como um dente anatomicamente normal na arcada, conforme achados de Atoche et al. (2014) e Granados (2020).

Já as anomalias de forma afetam as características normais da anatomia de um dente, seja de apenas uma parte do dente ou toda sua estrutura. Os dentes conóides são aqueles com formato anormal, de aparência cônica, e sua ocorrência varia de $1 \%$ a 16\% (Rodrigues et al., 2018; Giffoni, 2019); geminação ocorre quando não existe a separação completa do germe dentário dando origem a dois dentes, com prevalência de 0,08\% a 0,2\% (Negrete et al., 2015; Bilge et al., 2017); dens in dente se caracteriza por um dente dentro do outro, variando de 0,3\% a 8\% (Rodrigues et al., 2018; Giffoni, 2019); cúspide em garra é denominação para a formação de uma cúspide acessória na parte vestibular ou lingual, com variação de 1,04\% a 5,66\% (Pedreira, 2014; Rodrigues et al., 2018), e a taurodontia se apresenta como uma alteração típica da anatomia radicular na qual a câmara 
pulpar está alongada e canais radiculares estão curtos, enquanto clinicamente sua morfologia externa é normal, sendo demonstrada apenas por radiografia (Carvalho, 2011; Andrade et al., 2017; Bilge et al., 2017; Martins Neto et al., 2019).

As anomalias dentárias podem ser um desafio, já que causam problemas oclusais/funcionais, estéticos, com impactos já comprovados na qualidade de vida do paciente, uma vez que o sorriso desempenha papel importantíssimo no modo como o indivíduo se enxerga e como outros o enxergam (Pedreira, 2014). Devido às variações de anormalidades que podem ocorrer, em geral, o tratamento abrange mais de uma especialidade. Radiografias panorâmicas são uma ferramenta fundamental para o diagnóstico de anomalias, se apresentando, na maior parte das vezes, como um achado radiográfico, e não como motivo da procura odontológica, como é o caso de dentes supranumerários, que se encontram quase sempre inclusos e assintomáticos, não sendo diagnosticado ao exame clínico (Granados, 2020).

O conhecimento das anomalias dentárias é importante para o cirurgião-dentista e o diagnóstico precoce dessa condição pode modular o melhor prognóstico, podendo ser planejadas medidas preventivas que impeçam ou limitem suas eventuais consequências para a harmonia estético-funcional do sistema estomatognático (Najm, 2016). Os resultados das pesquisas que envolvem essas anomalias nem sempre são similares no que diz respeito a prevalência, e isso se deve a mudanças existentes entre técnicas de amostragem, critérios diferentes de diagnóstico, além das diferenças raciais existentes (Carneiro, 2014).

Considerando a maloclusão presente neste grupo de pacientes, é justificado que exista uma prevalência maior em pacientes ortodônticos (Magalhães et al., 2019). Sendo assim, o objetivo do presente trabalho foi avaliar a prevalência de anomalias de forma (taurodontia, conóide, dens in dente, geminação, cúspide em garra) e número (agenesia e supranumerário) em pacientes ortodônticos oriundos de uma clínica acadêmica de pós-graduação em Ortodontia situada em Santos, no estado de São Paulo (Brasil), visando colaborar com outros estudos populacionais e direcionar a atenção diagnóstica e o enfoque terapêutico na atuação de ortodontistas, reabilitadores orais e especialidades afins.

\section{Metodologia}

Este estudo observacional, transversal, seguiu todos os preceitos éticos da Resolução CNS 466/2012 e foi aprovado pelo Comitê de Ética em Pesquisa com parecer $\mathrm{n}^{\circ}$ 3.620.194. Todos os indivíduos assinaram o termo de consentimento livre e esclarecido concordando em participar do estudo. Foram analisadas 262 radiografias panorâmicas, de indivíduos do gênero masculino e feminino, com idade de 06 a 50 anos, que realizaram ou estão realizando tratamento ortodôntico. As radiografias examinadas pertencem ao banco de dados do curso de Especialização em Ortodontia da Faculdade de Odontologia da Universidade Metropolitana de Santos.

Para coleta de dados das anomalias dentárias, foi elaborada uma planilha no programa Microsoft Excel, com as seguintes informações: gênero, idade, clínica radiológica que realizou os exames, data de início do atendimento ortodôntico e anomalias de forma e número, e divididas em subseções de cada anomalia nas seguintes amostras: total, feminino e masculino, maxila e mandíbula.

As clínicas radiológicas que realizaram os exames foram planilhadas apenas para o caso de se necessitar alguma informação adicional relacionada à radiografia panorâmica ou ao modelo de estudo. Entretanto, considerou-se adequadas as radiografias selecionadas. Os laudos enviados pelo radiologista foram desprezados num primeiro momento, durante avaliação pela examinadora única, calibrada para realizar o diagnóstico de cada condição de anomalia, após aula expositiva e consulta bibliográfica indicada. Após o diagnóstico os laudos eram então consultados, para perícia. Havendo divergência entre a avaliadora e o laudo, um segundo avaliador era consultado, sendo sempre o orientador do trabalho.

Modelos de estudo confeccionados em gesso ou impressos digitalmente foram avaliados nos casos em que havia dúvidas referentes a alguma condição de anomalia. A avaliação da agenesia dos terceiros molares só foi incluída em pacientes acima dos 12 anos, tendo sido considerado como agenesia de terceiros molares apenas quando essa anomalia era detectada em pacientes 
com idade até 17 anos. A possibilidade de erros no diagnóstico de agenesias devidos à extrações terapêuticas dos $1^{\circ} \mathrm{s}$ ou $2^{\circ} \mathrm{s}$ prémolares, relacionadas ao tratamento ortodôntico, não pode ser excluída. Para minimizar este potencial erro se acrescentou a consulta dos prontuários clínicos à análise da radiografia panorâmica e de modelos.

A análise dos dados exigiu comparação de grupos não pareados para se verificar diferença entre as variáveis analisadas O teste não paramétrico de Mann-Whitney $(\mathrm{p}<0.05)$ foi usado para determinar se as observações de uma população tendem a ser maiores ou menores que outra população, homens/mulheres e maxila/mandíbula.

\section{Resultados}

Porcentagens e valores absolutos foram obtidos. Dentre as 262 radiografias panorâmicas observadas neste estudo, $41,98 \%$ eram do gênero masculino, enquanto $58,01 \%$ do gênero feminino, com a média de idade de 11,15 anos, como demonstrado na Tabela 1.

Tabela 1. Distribuição da amostra, por idade e gênero.*

\begin{tabular}{lcccccc}
\hline Idades & Masc. & $\%$ & Fem. & $\%$ & Total & $\%$ \\
\hline 06 a 10 & 6 & $2,29 \%$ & 8 & $3,05 \%$ & 14 & $5,34 \%$ \\
11 a 15 & 37 & $14,12 \%$ & 41 & $15,65 \%$ & 78 & $29,77 \%$ \\
16 a 20 & 21 & $8,01 \%$ & 22 & $8,40 \%$ & 43 & $16,41 \%$ \\
21 a 25 & 10 & $3,82 \%$ & 11 & $4,20 \%$ & 21 & $8,01 \%$ \\
26 a 30 & 9 & $3,43 \%$ & 18 & $6,87 \%$ & 27 & $10,30 \%$ \\
31 a 40 & 18 & $6,87 \%$ & 31 & $11,83 \%$ & 49 & $18,70 \%$ \\
41 a 50 & 9 & $3,43 \%$ & 21 & $8,01 \%$ & 30 & $11,45 \%$ \\
\hline Total & 110 & $41.98 \%$ & 152 & $58.01 \%$ & 262 & $100.00 \%$ \\
\hline
\end{tabular}

Foi observado um total de 114 anomalias dentárias, sendo a agenesia a anomalia mais frequente, se apresentando em 73 radiografias $(27,86 \%$ dos pacientes), sendo $30 \%$ apresentada apenas por terceiros molares conforme demonstrado no Gráfico 1, com maior ocorrência nas mulheres $n=42(57,5 \%) \times n=31(42,5 \%)$. O número e porcentagens das anomalias de número e forma estudadas se encontram listados na Tabela 2. Não houve diferença estatística entre os gêneros. O número e porcentagens de anomalias de número e forma, distribuídos de acordo com o gênero estão descritos na Tabela 3. Somente quando as agenesias envolveram especificamente ausência de dois elementos, em 18 pacientes (24,7\%), sendo 10 do gênero feminino e 8 do gênero masculino, se verificou prevalência de agenesias em mulheres, de modo significativo. 
Tabela 2. Número e porcentagens de anomalias de número e forma. *

\begin{tabular}{lcc}
\hline \multicolumn{1}{c}{ Anomalia } & $\mathbf{N}^{0}$ & $\%$ \\
\hline & & \\
Agenesia & 73 & $27.86 \%$ \\
Supranumerário & 11 & $4.20 \%$ \\
Mesiodens & 3 & $1.14 \%$ \\
Cúspide em Garra & 1 & $0.38 \%$ \\
Taurodontia & 19 & $7.25 \%$ \\
Dens in dente & 2 & $0.76 \%$ \\
Conóide & 2 & $0.76 \%$ \\
Geminação & 3 & $1.14 \%$ \\
\hline Total & 114 & \\
\hline \multicolumn{2}{c}{$*$ Fonte: Autores. }
\end{tabular}

Tabela 3. Número e porcentagens de anomalias de número e forma, distribuídos de acordo com o gênero. *

\begin{tabular}{lcccc}
\hline \multicolumn{1}{c}{ Anomalia } & Masc. & $\%$ & Fem. & $\%$ \\
\hline Agenesia & 31 & $42.46 \%$ & 42 & $57.53 \%$ \\
Supranumerário & 9 & $81.82 \%$ & 2 & $18.18 \%$ \\
Mesiodens & 2 & $66.67 \%$ & 1 & $33.33 \%$ \\
Cúspide em Garra & 0 & $0,00 \%$ & 1 & $100 \%$ \\
Taurodontia & 9 & $47.37 \%$ & 10 & $52.63 \%$ \\
Dens in dente & 1 & $50 \%$ & 1 & $50 \%$ \\
Conóide & 1 & $50 \%$ & 1 & $50 \%$ \\
Geminação & 1 & $33.33 \%$ & 2 & $66.67 \%$ \\
\hline Total & 54 & & 60 & \\
\hline & $*$ Fonte: Autores. & &
\end{tabular}


Gráfico 1. Porcentagens de agenesias encontradas incluindo os terceiros molares e descartando-os. *

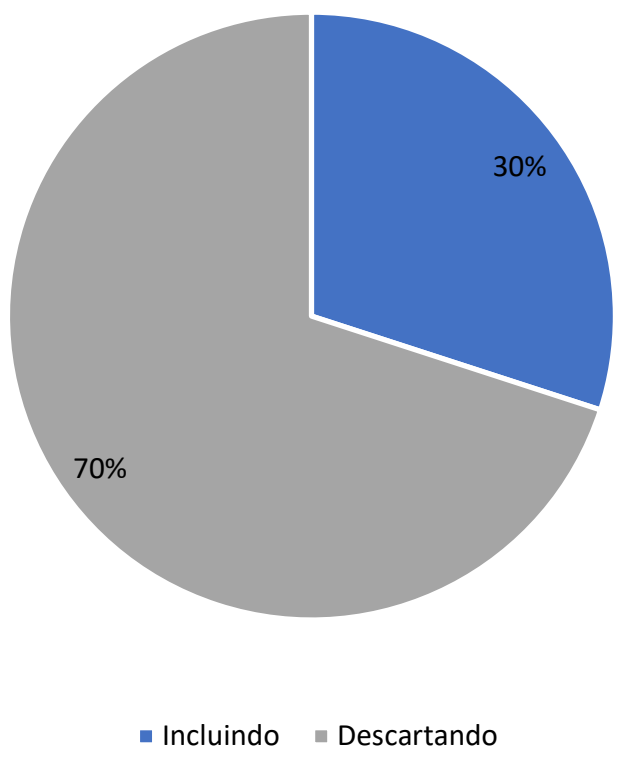

*Fonte: Autores.

Ao separar os pacientes em relação à quantidade de agenesia dentária, 52\% possuíam agenesia de somente um elemento e $48 \%$ agenesias de dois ou mais elementos. Separou-se as porcentagens das agenesias de um a 05 ou mais dentes, e se avaliou estatisticamente se os pacientes, tanto homens como mulheres, possuíam predominantemente agenesias unitárias ou múltiplas. Em mulheres, não houve diferença estatística na comparação entre os casos únicos de agenesia e os casos com múltiplas agenesias $(\mathrm{p}=0,121)$, mas nos homens se verificou superioridade da presença de agenesias únicas em cada paciente $(\mathrm{p}=0,042)$.

Anomalias de forma estiveram presentes em 111 (42,3\%) pacientes da amostra, divididos em 52 homens (46,8\%) e 59 mulheres $(53,2 \%)$, sem diferença estatística entre os gêneros, conforme o Quadro 1. Entretanto, quando se pretende verificar subgrupos relacionados à quantidade de anomalias de forma presentes em um mesmo paciente, e nesse caso, é estatisticamente significante a ocorrência de somente uma anomalia do que anomalias várias, tanto em homens como em mulheres. O Quadro 2 , abaixo, sintetiza os achados relacionados aos dentes envolvidos em anomalias de forma.

Quadro 1. Anomalias de forma, separadas por gênero e base óssea.*

\begin{tabular}{|l|c|c|c|c|c|c|}
\hline & 1 dente & 2 dentes & 3 dentes & 4 dentes & $5>$ dentes & total \\
\hline homens & $28(53,8 \%) \rightarrow * 14(26,9 \%)$ & $6(11,6 \%)$ & $3(5,8 \%)$ & $1(1,9 \%)$ & $52(46,8 \%)$ \\
\hline mulheres & $30(50,8 \%) \rightarrow * 17(28,8 \%)$ & $1(1,7 \%)$ & $3(5,1 \%)$ & $8(13,5 \%)$ & $59(53,2 \%)$ \\
\hline total & $58(52,3 \%) \rightarrow * 31(27,9 \%)$ & $7(6,3 \%)$ & $6(5,4 \%)$ & $9(8,1 \%)$ & $111(100 \%)$ \\
\hline
\end{tabular}

*Fonte: Autores.

Quadro 2. Envolvimento de elementos dentários em anomalias de forma.*

\begin{tabular}{|l|c|c|c|c|c|}
\hline & Número dentes & homens & mulheres & Maxila & mandíbula \\
\hline Conoide & 2 & 1 & 1 & 2 & 0 \\
\hline Em garra & 1 & 0 & 1 & 1 & 0 \\
\hline Dens in dent & 2 & 1 & 1 & 2 & 0 \\
\hline Geminação & 3 & 1 & 2 & 2 & 1 \\
\hline Taurodontia & 19 & 9 & 10 & $12 \longrightarrow 26$ \\
\hline
\end{tabular}

*Fonte: Autores. 
A taurodontia é a segunda anomalia mais frequente apresentada por 7,25\% dos casos, sendo $47,37 \%$ do gênero masculino e 52,63\% do gênero feminino, sem diferença estatística entre os gêneros. O taurodontismo também é a anomalia de forma que mais envolveu elementos dentários (19), divididos sem diferença significante entre homens e mulheres (9 x 10), tendo sido, entretanto, mais presente em mandíbula que em maxila, de modo significativo $(\mathrm{p}=0,0029)$. As demais anomalias de forma apareceram igualmente entre homens e mulheres, entre maxila e mandíbula.

Supranumerários foram contabilizados em 11 pacientes $(4,20 \%)$, ocorrendo mais em homens $n=9 ;(81,8 \%)$ do que em mulheres $n=2 ;(18,2 \%)$, com diferença estatística entre os gêneros nessa amostra $(p=0,003)$. Na comparação entre as bases ósseas, houve diferença estatística com maior ocorrência registrada na maxila do que na mandíbula.

O Quadro 3 sintetiza a ocorrência de todas as anomalias estudadas, quantificando-as em número e porcentagens, e separados por sua ocorrência em maxila ou mandíbula.

Quadro 3. Número e porcentagens das anomalias encontradas, separadas em mandíbula e maxila.*

\begin{tabular}{|l|c|c|c|c|}
\hline & Maxila & $\%$ & Mandíbula & $\%$ \\
\hline Agenesia & 84 & $56 \%$ & 66 & $44 \%$ \\
\hline Supranumerário & 13 & $86.67 \%$ & 2 & $13.33 \%$ \\
\hline Cúspide em Garra & 2 & $100 \%$ & 0 & $0,00 \%$ \\
\hline Taurodontia & 12 & $31.58 \%$ & 26 & $68.42 \%$ \\
\hline Dens in dente & 2 & $100 \%$ & 0 & $0,00 \%$ \\
\hline Conoide & 2 & $100 \%$ & 0 & $0,00 \%$ \\
\hline Geminação & 1 & $33.33 \%$ & 2 & $66.67 \%$ \\
\hline \multicolumn{1}{|c|}{ Total } & 116 & & 96 & \\
\hline
\end{tabular}

*Fonte: Autores.

Quadro 4. Envolvimento de elementos dentários supranumerários.*

\begin{tabular}{|c|c|c|c|c|}
\hline & 1 dente & 2 dentes & 3 dentes & total \\
\hline homens & $4(44,4 \%)$ & $4(44,4 \%)$ & $1(11,2 \%)$ & $9(81,8 \%)$ \\
\hline mulheres & 0 & $2(100 \%)$ & 0 & $2(18,2 \%) * \checkmark$ \\
\hline total & $4(36,3 \%)$ & $6(54,6 \%)$ & $1(9,1 \%)$ & $11(100 \%)$ \\
\hline
\end{tabular}

*Fonte: Autores.

\section{Discussão}

Sabendo da existência de anomalias dentárias com frequência e variabilidade distintas entre as populações verificadas, se torna de suma importância seguir ampliando o estudo das prevalências, visando contribuir com a Ciência, à medida que se pode aperfeiçoar sua identificação para que o cirurgião-dentista consiga realizar um diagnóstico cada vez mais precoce, atuando adequadamente sobre a diversidade de problemas clínicos que essas anomalias podem causar (Bilge et al., 2017). Por serem assintomáticas, as radiografias panorâmicas - que tradicionalmente constituem as documentações dos pacientes - são importantes meios de diagnóstico de anomalias (Jiménez-Sánchez, 2019). Dentre as 262 radiografias panorâmicas observadas na presente pesquisa foram encontradas um total de 114 anomalias dentárias. Esse alto índice provavelmente se justifica pela própria especialidade para a qual tais pacientes foram selecionados, considerando que indivíduos com anomalias dentárias podem apresentar-se com complicações no processo normal de oclusão, levando-os a busca por tratamento ortodôntico (Magalhães et al., 2019).

A anomalia mais frequente observada neste estudo foi a agenesia $(27,86 \%)$, em concordância com a literatura, onde Carneiro (2014) encontrou 64,2\% de ocorrência na capital de Mato Grosso do Sul, Campo Grande, 33,4\% na amostra da 
Universidade Autônoma de Yucatan (México) (Herrera-Atoche et al., 2014) e 12,2\% na população portuguesa, a partir de estudo na Universidade do Porto (Carvalho et al., 2011). Entretanto, houve trabalhos, de diferentes populações, que divergiram dessa prevalência, obtendo porcentagem maior de dentes impactados (13,5\%) (Pedreira, 2014), ectopia (35,0\%) (Jiménez-Sánchez, 2019); dentes não irrompidos (41,7\%) (Martins Neto et al., 2019) e anomalias de posição (60,8\%) (Bilge et al., 2017), reforçando a constatação de alta variabilidade populacional.

Jiménez-Sánchez et al. (2019) demonstraram que a prevalência de agenesia é de 33,4\% em pacientes que acudiram a um centro radiológico em Guadalajara, México. Entretanto, cai para somente 4,87\% ao se excluir terceiros molares da amostra. Os terceiros molares são os dentes mais ausentes, seguidos pelos pré-molares e incisivos respectivamente. Carter et al. (2015) defenderam que a alta prevalência de agenesia de terceiros molares ocorre por ser o último dente a se desenvolver e erupcionar no arco dentário, sendo assim, mais susceptível a perturbações ambientais. A agenesia, na presente pesquisa, diferiu bastante na quantificação da prevalência, com 30\% incluindo os terceiros, e 70\% excluindo-os, o que pode ser atribuído à metodologia empregada, onde se interpretou que havia agenesia de terceiros molares quando a ausência foi verificada a partir dos 12 anos, para minimizar erros no diagnóstico, devido dificuldades de distinguir se há agenesias ou se esses dentes ainda estariam em desenvolvimento em idades mais precoces. Metodologicamente, também foi definido critério para classificar agenesia dos terceiros molares quando ausentes apenas até os 17 anos de idade, considerando a alta quantidade de extrações durante a adolescência. Logo, se tais dentes não foram encontrados nos pacientes adultos não se considerou que tais pacientes possuíam agenesia de terceiros molares, devido a alta ocorrência de extrações desses elementos durante fim do crescimento; dado que indica exatamente porque terceiros molares extraídos não se relacionaram com o conceito de agenesia nesse trabalho.

Nota-se que na literatura já se obteve muitos resultados semelhantes ao encontrado nesse estudo, a exemplo dos achados mexicanos de Herrera-Atoche et al. (2014), nos quais os dentes mais ausentes são os segundos pré-molares (25,7\%) seguido dos incisivos laterais inferiores (22,7\%); de trabalho desenvolvido por Najm et al. (2016) na Universidade de Bagdá (Iraque), com ausência dos segundos pré-molares inferiores (16\%); da Universidade da Jordânia (Amã) com os incisivos laterais superiores $(87,7 \%)$ seguido dos segundos pré-molares inferiores (64,3\%) (Al-Abdallah et al.,2015), de New South Wales (Austrália) com os segundos pré-molares inferiores (42,0\%) seguido dos incisivos laterais superiores (27,5\%) (Dang et al., 2017), e de Santiago do Chile com segundos pré-molares inferiores $(34,1 \%)$ seguido dos incisivos laterais superiores $(23,6 \%)$, segundo Diaz et al. (2020).

Todavia, outros trabalhos relataram prevalência maior quando terceiros molares são incluídos na amostra, como Carneiro (2014) em Campo Grande (MS), onde o maior acometimento de agenesias tem relação com os elementos 18 (20,1\%), 28 (19,9\%), 38 (16,9\%) e 48 (15,6\%). Na cidade do Porto foi verificado 12,2\% de agenesias com inclusão dos terceiros molares, e 6,47\%, excluindo-os (Carvalho et al., 2011). O estudo da Universidade Autônoma de Yucatan (México) verificou 33,4\% de agenesias com inclusão dos terceiros molares, e 4,87\%, excluindo-os (Herrera-Atoche et al., 2014). Deve-se considerar também que alguns trabalhos os descartaram complemente em suas pesquisas, a exemplo de Freitas et al. (2012), Pedreira (2014), Torres et al. (2015), Yunis et al. (2017), Capoani e Gonçalves (2019) e Magalhães et al. (2019).

Em síntese, se observou na literatura que, quando não se envolviam terceiros molares, os dentes mais acometidos pelas agenesias foram os segundos pré-molares do arco mandibular (Yunis et al., 2017; Capoani e Gonçalves, 2019; Giffoni et al., 2019). No arco maxilar, a maior ocorrência de agenesias foi dos incisivos laterais, exceto no trabalho de Yunis et al. (2017), que verificaram maior acometimento dos pré-molares no arco maxilar. A maior ocorrência de agenesia de incisivo lateral superior foi bilateral. Quando seu aparecimento se dá de forma unilateral, seu contralateral costuma se encontrar associado à microdontia, uma anomalia de tamanho (Herrera-Atoche et al., 2014). O gênero feminino foi o mais afetado (Carvalho et al., 2011), numa proporção de 10:7, estando de acordo com o presente estudo, no qual verificamos agenesia em 18 pacientes (24,7\%), sendo 10 do gênero feminino e 8 do gênero masculino, dado que pode estar relacionada a uma maior procura por mulheres de atendimento 
na clínica de Ortodontia.

Em relação à quantidade de agenesia dentária, em mulheres, não houve diferença estatística na comparação entre os casos únicos de agenesia e os casos de múltiplas agenesias ( $\mathrm{p}=0,121)$, mas nos homens foi apontado superioridade da presença de agenesias únicas em cada paciente ( $\mathrm{p}=0,042$ ), estando de acordo com Bilge et al. (2018), que verificou que 90\% de anomalias foram únicas.

Em nosso estudo a taurodontia se apresentou como a segunda anomalia mais frequente $(7,25 \%)$, diferente dos achados de Najm et al. (2016), que encontraram a presença de incisivo lateral conóide como anomalia prevalente (16\%) em estudantes de odontologia iraquianos, com taurodontia aparecendo por último (4\%) na amostra. Nossa pesquisa se contrapôs também com trabalhos realizados em Alfenas (MG), com 4,98\%, São José do Rio Preto $(0,78 \%)$, Teresina $(0,66 \%)$ e em cidades cearenses $(0,3 \%)$. (Pedreira, 2014; Negrete et al., 2015; Torres et al., 2015, e Martins Neto et al., 2019, respectivamente).

A geminação apresentou-se em $1,14 \%$ em nossa amostra, havendo porcentagens ainda mais baixas verificadas por Negrete et al. (2015) em São José do Rio Preto (0,2\%) e por Bilge et al. (2018), na Turquia (0,08\%).

Os dentes conóides apareceram apenas em $0,76 \%$ em nosso trabalho, e essas baixas ocorrências também foram encontradas nas amostras de Torres et al., 2015 (1,0\%) e de Herrera-Atoche et al., 2014 (1,49\%). Estudo iraquiano, por outro lado, encontrou prevalência de 16\% de dentes conóides (Najm et al, 2016).

E assim como os dentes conóides, os dens in dente foram encontrados com porcentagem de $0,76 \%$, com expressão semelhante na tese de Carneiro (2014), divergindo novamente do trabalho iraquiano (Najm et al, 2016).

A anomalia com menor prevalência, dentre as pesquisadas, foi de dentes com cúspide em garra $(0,38 \%)$. Contudo, pesquisa piauiense encontrou 5,6\% (Torres et al., 2015).

Os dentes supranumerários corresponderam a 4,2\% -- resultados próximos foram obtidos em Teresina - PI (4,0\%)' por Torres et al. (2015), e em estudo universitário no Rio Grande do Sul, com 4,67\%, por Capoani e Gonçalves (2019). Os supranumerários são mais encontrados na maxila, em região anterior, sendo chamados de mesiodens (Capoani, Gonçalves; 2019; Magalhães et al., 2019). Resultados diferentes foram verificados por Torres et al. (2015), que destacaram o quarto molar como supranumerário mais frequente. O gênero masculino foi o mais afetado pela ocorrência de supranumerários. Pedreira (2014) obteve maior prevalência de supranumerários na maxila, diferente de Torres et al. (2015) que não verificaram diferença nos supranumerários, justamente o grupo onde encontramos diferença estatística.

Portanto, exceto pelos supranumerários, mais presentes na maxila, e pela taurodontia, que esteve mais presente em mandíbula, esse trabalho não encontrou diferença estatisticamente significativa entre as bases ósseas maxila e mandíbula na prevalência das anomalias. Na literatura, anomalias em geral são relatadas com maior prevalência no gênero feminino (Carneiro, 2014; Al-Abdallah et al., 2015; Giffoni et al. 2019), porém, não encontramos diferenças estatísticas em nossa pesquisa em relação ao gênero, exceto pela diferença para mulheres na ocorrência de agenesia apenas quando envolvem ausência de dois elementos dentários, especificamente. Podemos ainda verificar diferença estatisticamente significativa entre os gêneros no caso dos supranumerários, prevalecendo em homens $(81,8 \%)$ em relação às mulheres, como demonstrado no quadro 4.

As maloclusões parecem estar relacionadas às anomalias dentárias, e as próprias anomalias podem estar associadas entre si, resultando em alterações oclusais, como sobremordida profunda, Classe II e Classe III de Angle, dentre outras, e por isso merecem toda atenção necessária para prevenção e interceptação de maloclusões em pacientes com anomalias de número e forma.

\section{Conclusão}

Após avaliação descritiva pode-se concluir que as anomalias de número foram muito mais prevalentes do que as anomalias de forma, sendo agenesia e taurodontia as anomalias mais prevalentes em pacientes ortodônticos da Clínica de 
Especialização em Ortodontia da Universidade Metropolitana de Santos, em São Paulo.

Sugere-se que pesquisas futuras possam unificar os dados de centros acadêmicos e clínicas públicas e privadas de toda uma cidade ou região para propor um levantamento epidemiológico que pudesse guiar os profissionais ortodontistas e reabilitadores em suas ênfases terapêuticas. Diferenças étnicas devem, de igual modo, ser investigadas.

\section{Referências}

Al-Abdallah, M., AlHadidi, A., Hammad, M., Al-Ahmad, H. \& Saleh, R. (2015) Prevalence and distribution of dental anomalies: A comparison between maxillary and mandibular tooth agenesis. Am J Orthod Dentofacial Orthop. 148: 793-98.

Andrade, C.E.S., Lima, I.H.L., Silva, I.V.S., Vasconcelos, M.G. \& Vasconcelos, R.G. (2017). As principais alterações dentárias de desenvolvimento. Rev Salusvita (Online).36 (2): 533-63.

Atoche, J.R.H., Morales, S.M.D., Ruiz, G.E.C., Ramírez, M.E. \& Orellana, M.F. (2014). Prevalence of dental anomalies in a Mexican population. Dentistry 3000. 2(1): $1-5$

Bilge, N.H., Yeşiltepe, S., Ağırman, K.T., Çağlayan, F. \& Bilge, O.M. (2017) Investigation of prevalence of dental anomalies by using digital panoramic radiographs. Folia Morphol. 77 (2): 323-28.

Capoani, V. \& Gonçalves, A.L.C.A. (2019) Avaliação da prevalência de agenesia de incisivos laterais superiores dos pacientes da Faculdade de Odontologia do Centro Universitário da Serra Gaúcha. J of Oral Invest. 8 (1): 57-68.

Carneiro, G.V. (2014). Estudo radiográfico da prevalência de anomalias dentárias por meio de radiografias panorâmicas em diferentes faixas etárias [Tese]. Campo Grande (MS): Programa de Pós-Graduação em Saúde e Desenvolvimento, Universidade Federal de Mato Grosso do Sul.

Carter, K. \& Worthington, S. (2015). Morphologic and Demographic Predictors of Third Molar Agenesis: A Systematic Review and Meta-analysis. J Dent Res. $1-9$.

Carvalho, S., Mesquita, P. \& Afonso, A. (2011). Prevalência das anomalias de número numa população portuguesa. Estudo radiográfico. Rev Port Estomatol Med Dent Cir Maxilofac. 52 (1): 7-12.

Dang, H. Q., Constantine, S., \& Anderson, P. J. (2017). The prevalence of dental anomalies in an Australian population. Australian dental journal, 62(2), 16164. https://doi.org/10.1111/adj.12443.

Diaz, K.Y, Escobar, I.R, Torres, N.A, Monsalve, V.R., \& Soler, C.T. (2020). Prevalência de agenesia dentária em pacientes avaliados em ortodontia, Santiago do Chile. Vital Dentistry. 32 (1): 57-62.

Fournier, B. P., Bruneau, M. H., Toupenay, S., Kerner, S., Berdal, A., Cormier-Daire, V... de La Dure-Molla, M. (2018). Patterns of Dental Agenesis Highlight the Nature of the Causative Mutated Genes. Journal of dental research. 97(12), 1306-316. https://doi.org/10.1177/0022034518777460.

Freitas, D.Q, Tsumurai, R.Y \& Filho Machado, D.N.S.P. (2012). Prevalence of dental anomalies of number, size, shape and structure. Rev Gauch Odontol. 60 (4): 437-41.

Giffoni, T.C.R., Brandt, G.Z., Rocha, I.S., Ramos, A.L., Provenzano, M.G.A. \& Fracasso, M.L.C. (2019) Relation of Dental Anomalies with Occlusal Alterations in the Pediatric Patients. Pesqui Bras Odontopediatria Clín Integr. 19:e4026.

Granados, J.K.Z. (2020). Prevalencia de anomalías dentarias en niños con dentición permanente [Tese]. Guayaquil (Ecuador): Facultad de Odontología, Universidad de Guayaquil.

Grau, W.G. (2016). Achados incidentais em radiografias panorâmicas de pacientes pré e pós-tratamento ortodôntico [Dissertação]. Bauru (SP), Faculdade de Odontologia de Bauru, Universidade de São Paulo.

Jiménez-Sánchez, A.C. \& Sierra-Robles, E. (2019). Frecuencia de agenesias dentales en pacientes que acudieron a un centro radiológico en Guadalajara, México. Rev Tamé. 7. 8(22): 866-69.

Magalhães, G.P., Paz, E.C., Sousa, Y.T.C.S., Leite, C.M.C., Falcão, C.A.M. \& Ferraz, M.A.A.L. (2019) Diagnóstico de anomalias dentárias em radiografias panorâmicas. Rev Odontol Bras Central. 28 (87): 244-47.

Martins Neto, R. S., de Sousa Alves, I. F., Machado, A. L., Barbosa Neto, L. A., Alencar, A. A., \& Esses, D. F. S. (2019). Prevalência de anomalias dentárias em radiografias panorâmicas. Arch Health Invest. 8 (2):68-73.

Najm, A.A., Mahdi, A.S., Al-Sudani, R.J. \& Musa, H.H. (2016). Prevalence of Dental Anomalies among Iraqi Dental Students. J Bagh Coll Dentistry. 28 (4):726.

Negrete, D., Carvalho, P.E.G., Fuziy, A., Torres, F.C., Triviño, T. \& Flaiban, E. (2015). Prevalência de dentes supranumerários em pacientes de Ortodontia. Rev Odontol Univ Cid São Paulo. 27 (1): 6-13. 
Research, Society and Development, v. 10, n. 9, e3910917504, 2021

(CC BY 4.0) | ISSN 2525-3409 | DOI: http://dx.doi.org/10.33448/rsd-v10i9.17504

Pedreira F.R.O. (2014). Prevalência de Anomalias Dentárias em uma população ortodôntica na região de Alfenas/MG [Dissertação]. Alfenas (MG): Programa de Pós-graduação em Ciências Odontológicas, Universidade Federal de Alfenas.

Rodrigues, A.S., Freire, J.S., Silva, G.I.M., Antunes, L.S. \& Antunes, L.A.A. (2018). Does dental agenesis have an impact on OHRQoL of children, adolescents and young adults? A systematic review. Acta Odontol Scand.1-7.

Santos, K.S.S., Correia Neto, I.J., Soares, I.S., Araújo, L.M., Nascimento, F.S., Rebelo, H.L. Franco, A.V.M. (2017). Anomalias dentárias simultâneas: Relato de caso. RvAcBO. 7 (2):131-34.

Silvério, P.N., Osório, S.G., Osório, A., \& Queiroz, P.M. (2019). Estudo epidemiológico de dentes supranumerários diagnosticados pela radiografia panorâmica. Rev Uningá Maringá.56(S5): 115-21.

Torres, P. F., Simplício, A. H. M., Luz, A. R. C. A., Lima, M. D. M., Moura, L. F. A. D., \& Moura, M. S. Anomalias dentárias de número em pacientes ortodônticos. Rev Odontol UNESP. 2015; 44(5): 280-284.

Yunis, L. L. de M., Bruzadin, L. N., Martin, L. G., Bruzadin, L. N., Silva, V. S. da, Cruz, M. C. C., Moreti, L. C. T. (2018). P o69 - Estudo radiográfico da prevalência de anomalias dentárias de número através de radiografias panorâmicas de uma clínica odontológica. Archives of Health Investigation, 6 . Recuperado de https://www.archhealthinvestigation.com.br/ArcHI/article/view/2884. 